\title{
A comparative structural analysis reveals distinctive features of co-factor binding and substrate specificity in plant aldo-keto reductases
}

Priscila Oliveira de Giuseppe ${ }^{1, *}$, Marcelo Leite dos Santos ${ }^{2, *,}$, Sylvia Morais de Sousa $^{3}$, Karen E. Koch ${ }^{4}$, José Andrés Yunes ${ }^{5,6}$, Ricardo Aparicio ${ }^{2, \S}$, Mario Tyago Murakami $^{1, \S}$

${ }^{1}$ Biosciences National Laboratory (LNBio), National Center for Research in Energy and Materials (CNPEM), Campinas, SP, Brazil.

${ }^{2}$ Laboratory of Structural Biology and Crystallography, Institute of Chemistry, University of Campinas, Campinas, SP, Brazil.

${ }^{3}$ Embrapa Maize and Sorghum, Sete Lagoas, MG, Brazil.

${ }^{4}$ Department of Horticultural Science, University of Florida, Gainesville, FL, USA.

${ }^{5}$ Centro Infantil Boldrini, Campinas, SP, Brazil.

${ }^{6}$ Department of Medical Genetics, Faculty of Medical Sciences, University of Campinas, Campinas, SP, Brazil.

*Both authors contributed equally to this work

${ }^{¥}$ Current address: Centre of Exact Sciences and Technology, Federal University of Sergipe, Itabaiana, SE, Brazil.

${ }^{\S}$ Corresponding authors: Ricardo Aparicio (aparicio@iqm.unicamp.br) and Mario T. Murakami (email: mario.murakami@lnbio.cnpem.br; Fax: 55-19-3512-1004; Tel: 5519-3512-1106) 


\begin{abstract}
Plant aldo-keto reductases of the AKR4C subfamily play key roles during stress and are attractive targets for developing stress-tolerant crops. However, these AKR4Cs show little to no activity with previously-envisioned sugar substrates. We hypothesized a structural basis for the distinctive cofactor binding and substrate specificity of these plant enzymes. To test this, we solved the crystal structure of a novel AKR4C subfamily member, the AKR4C7 from maize, in the apo form and in complex with NADP ${ }^{+}$. The binary complex revealed an intermediate state of cofactor binding that preceded closure of Loop B, and also indicated that conformational changes upon substrate binding are required to induce a catalytically-favorable conformation of the active-site pocket. Comparative structural analyses of homologues (AKR1B1, AKR4C8 and AKR4C9) showed that evolutionary redesign of plant AKR4Cs weakened interactions that stabilize the closed conformation of Loop B. This in turn decreased cofactor affinity and altered configuration of the substrate-binding site. We propose that these structural modifications contribute to impairment of sugar reductase activity in favour of other substrates in the plant AKR4C subgroup, and that catalysis involves a three-step process relevant to other AKRs.
\end{abstract}

Keywords: plant aldose reductase, AKR4C subfamily, crystal structure, intermediate state, cofactor binding. 


\section{Introduction}

The aldo-keto reductases (AKRs) are $\mathrm{NAD}(\mathrm{P}) \mathrm{H}$-dependent enzymes that reduce a wide variety of carbonyl substrates such as sugar aldehydes, keto-steroids and lipid peroxidation by-products. In humans, they have been implicated in diabetic complications and congenital diseases [1], while in plants they play a key role in reactive aldehyde detoxification, osmolite production, and secondary metabolite synthesis [2].

Members of the AKR superfamily are classified into 16 families based on amino acid sequence identity [1]. Members of families 1, 6 and 7 are found in humans and other mammals, whereas the majority of AKRs studied in plants belong to family 4 [1, 2]. Family 1 shows a broad substrate specificity and includes the closest mammalian homologue of plant-family $4[2,3]$. Based on substrate preference, family 1 is further subdivided into five subfamilies: (A) aldehyde reductases; (B) aldose reductases; (C)

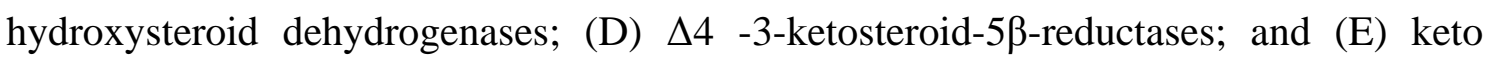
reductases [1]. From these enzymes, the aldose reductase AKR1B1, which converts glucose to sorbitol, has been extensively studied via structural approaches. Much of this earlier work was aimed at understanding the binding mode of specific inhibitors employed to prevent diabetic complications [4].

The AKR family 4 includes three subfamilies (A, B and C), which have been implicated in (A) plant-microbe interactions, (B) iron acquisition from soil, and (C) stress defense [2, 3]. In particular, members of subfamily $4 \mathrm{C}$ have emerged as promising targets for generation of plants tolerant to multiple abiotic stresses [5]. To date, only three members of subfamily $4 \mathrm{C}$ have been structurally characterized: AKR4C1 [6], AKR4C8 and AKR4C9 [7]. The AKR4C1 is an embryo-specific enzyme associated with desiccation tolerance in barley [8], whereas AKR4C8 and AKR4C9 are 
upregulated in Arabidopsis leaves under stress conditions such as cold, salinity and drought [7].

Members of the AKR4C subfamily have been implicated in aiding desiccation tolerance based on their capacity to convert glucose into the osmolyte sorbitol $[2,8]$. However, in vitro studies show that these enzymes are poorly active or even inactive with glucose $[7,9,10]$. Although the structural basis for the substrate preferences of some AKR4C enzymes is known [6,7], the molecular adaptations that impair their function on osmoregulatory-relevant substrates, such as glucose, remain elusive. Moreover, the lack of high-resolution structures for the binary enzyme $\operatorname{NADP}(\mathrm{H})$ complexes, without the influence of ligands in the substrate-binding site, prevents further insights into conformational changes of AKR4C enzymes during intermediate steps of the catalytic cycle.

We therefore tested the hypothesis that structural features of intermediate steps in catalysis were central to the reduced affinity of plant AKR4Cs for sugar substrates. Toward this end, we solved the crystal structure of a novel member of the subfamily $4 \mathrm{C}$ from Zea mays (AKR4C7) in the apo form and in complex with NADP ${ }^{+}$. Our data reveal the formation of an intermediate state of the complex with $\mathrm{NADP}^{+}$and indicate that substrate binding induces a catalytically-favorable conformation of the active-site pocket. In addition, our comparative analyses support the proposal that structural adaptations of the plant AKR4Cs provide a mechanistic basis for the observed impairment of glucose-reductase activity in this subfamily. The relevance of these findings extend to other plant AKRs that play key roles in stress and defense, since AKR4C7 shares about 70\% sequence identity with AKR4C9 and AKR4C8. 


\section{Materials and Methods}

\section{Protein overexpression, purification and crystallization}

Recombinant AKR4C7 (UNIPROT ID: A2T1W7) fused to an N-terminal Histag was overexpressed in Escherichia coli BL21(DE3), purified by metal-affinity and anion-exchange chromatographies [10], and crystallized by vapour diffusion as described by Kiyota and co-workers [11]. The AKR4C7•NADP ${ }^{+}$complex was cocrystallized in a condition similar to that of the apoenzyme $(0,1 \mathrm{M}$ Tris- $\mathrm{HCl}, \mathrm{pH}, 6.0$, $22 \% \mathrm{~m} / \mathrm{v}$ PEG4000 and 0,1 M sodium acetate) pre-incubating AKR4C7 at $9 \mathrm{mg} / \mathrm{ml}$ with 10 mM NADP ${ }^{+}$

$X$-ray diffraction data collection, processing and structure determination

For data collection, crystals were soaked in a cryoprotectant solution containing $15 \%(v / v)$ ethylene glycol and flash-cooled in a nitrogen stream at $100 \mathrm{~K}$ (Oxford Cryosystems). Data were collected by the oscillation method at the MX2 beamline from LNLS (Campinas, Brazil) using a Marmosaic-225 CCD detector (MarUSA) and setting the X-ray wavelength to $1.458 \AA$. Data were indexed and integrated using the MOSFLM program [12] and reduced using the SCALA program [13] from the CCP4 package [14]. The crystal structure of AKR4C7 (apoenzyme) was solved by molecular replacement using the MrBUMP program [15] from $C C P 4$ and the structure of pig aldose reductase (PDB ID: 1EKO) as template [16]. The refined structure of the apo AKR4C7 was used as input in the AMORE program [17] to solve the crystal structure of the AKR4C7 $\cdot \mathrm{NADP}^{+}$complex by molecular replacement. Crystallographic structure was refined using alternating cycles of REFMAC5 [18] with visual inspection of the 
electron density maps and manual rebuilding with COOT [19]. The stereochemistry of the model was analyzed with Molprobity [20]. Statistics of data collection and model refinement are summarized in Table 1. Figures were produced using Pymol [21].

\section{Results and Discussion}

The active site of both apo- and NADP+-bound AKR4C7 show an open conformation with local disorders

The crystal structures of AKR4C7 in the apo form and in complex with $\mathrm{NADP}^{+}$ were solved at 1.45 and $1.72 \AA$ resolution, respectively (Table 1). They revealed that AKR4C7 has the typical $(\alpha / \beta)_{8}$-barrel fold of the aldose reductase superfamily and preserves the catalytic tetrad Asp ${ }^{44}, \mathrm{Tyr}^{49}, \mathrm{Lys}^{78}$ and His ${ }^{111}$ characteristic of AKRs [22]. In both crystal structures, regions from Loop A $\left(\mathrm{Arg}^{115}-\mathrm{Asp}^{131} / \mathrm{Phe}^{132}\right)$ and Loop C $\left(\mathrm{Ala}^{284}-\mathrm{Leu}^{310}\right)$ were disordered (Fig. 1). The Loop B was also partially disordered $\left(\mathrm{Gly}^{206}-\mathrm{Gly}^{215}\right)$ in the AKR4C7-NADP ${ }^{+}$complex, but it showed a well-structured, open conformation in the apo form (Fig. 1).

$<$ Figure $1>$

The Loops A, B and C define the shape and the plasticity of the AKR active site, playing a role in co-factor/substrate binding and substrate specificity [6, 7]. Comparison of the apo form of aldose reductases from different species reveals diverse sizes and conformations for these loops, especially Loop B (Fig. S1A). The single apo structure known for plant AKRs thus far is the apo-AKR4C1 from barley (PDB ID: 2BGQ). This structure shares $44 \%$ sequence identity with AKR4C7 and can be superimposed into apo-AKR4C7 with an RMSD of $1.26 \AA$ for $248 \mathrm{C} \alpha$ atoms aligned. In contrast with 
AKR4C7, Loops A and C from the barley AKR4C1 are folded, whereas Loop B is partially disordered (Fig. S1B).

Comparative analysis of human AKR1B1 in the apo- and cofactor-bound forms shows similar arrangements for Loops A and C, but a large conformational change in Loop B upon NADP(H) binding [23]. These appraisals indicate that $\mathrm{NADP}(\mathrm{H})$ induces Loop B to adopt a closed conformation that fastens this cofactor into the active site. In contrast, our data show that, for the plant $\mathrm{AKR} 4 \mathrm{C} 7$, cofactor binding disturbs the open conformation of Loop B, but is insufficient to induce closure of this loop (Fig. 1). Supporting this interpretation is the observation that the nicotinamide nucleotide moiety of the cofactor as well as the Gly ${ }^{206}-\mathrm{Gly}^{215}$ segment of Loop B were disordered in the $\mathrm{AKR} 4 \mathrm{C} 7 \cdot \mathrm{NADP}^{+}$complex. This disorder indicates an intermediate state of cofactor binding (Fig. 1B). Our data agree with kinetic studies of rat liver AKR1C9 that suggest NADPH binding occurs in three steps: 1) the fast formation of a loose complex $(E \bullet \mathrm{NADPH})$ followed by two conformational changes of the enzyme leading to 2$)$ an intermediate $\left(E^{*} \cdot \mathrm{NADPH}\right)$ and 3$)$ to a tightly bound complex $\left(E^{* *} \cdot \mathrm{NADPH}\right)$ [24]. In this context, our results support a model in which the formation of an intermediate $E^{*} \cdot \mathrm{NADPH}$ complex anchors the adenosine $2^{\prime}, 5^{\prime}$-diphosphate moiety and consequently increases flexibility of Loop B.

To further define structural components of this hypothesis, we focused on conformational changes involved in the transition from the intermediate to the tightlybound complex between AKR4C7 and NADP(H). A search for homologous structures in the Protein Data Bank using PDBeFold software [25] led to AKR4C9 of Arabidopsis thaliana, which has a 74\% sequence identity to AKR4C7. A superimposition of these two AKRs gave an RMSD of $1.08 \AA$ for $255 \mathrm{C} \alpha$ atoms aligned. The crystal structure of AKR4C9, which comprises the ternary complex $E \bullet N^{\prime} D^{+} \bullet$ acetate (PDB ID: $3 H 7 U$ ) 
[7], reveals a closed Loop B and a well-ordered co-factor, thus reflecting a tightly bound complex. The AKR4C7 and AKR4C9 also share the same cofactor-binding site, except for the conservative amino acid substitutions T233S, Q261E and L287V. We thus undertook a comparative analysis of the AKR4C7•NADP ${ }^{+}$and AKR4C9 $\bullet \mathrm{NADP}^{+} \cdot$ acetate structures to determine what aspects of their conformation could lead the intermediate complex between AKR4C7 and $\mathrm{NADP}(\mathrm{H})$ to progress into a tightly-bound state (Fig. 2).

\section{$<$ Figure $2>$}

This comparison revealed several changes in the catalytic interface involving not only the loops $\mathrm{A}, \mathrm{B}$ and $\mathrm{C}$, but also the $\beta_{1}-\alpha_{1}$ loop $\left(\operatorname{Ser}^{15}-\mathrm{Gly}^{26}\right)$. In the AKR4C7 $\bullet \mathrm{NADP}^{+}$structure, the $\operatorname{Trp}^{21}$ residue assumes a position that orients Lys ${ }^{252}$ towards the active site. This in turn disturbs anchoring of the 5'-phosphoryl group of the nicotinamide nucleotide moiety and creates a pocket where a 1,2-ethanediol molecule is bound (Fig. 2A). According to the AKR4C9 structure, once Loop B moves to the closed conformation, its $\operatorname{Trp}^{212^{*}}$ residue attracts $\operatorname{Trp}^{21^{*}}$, inducing a conformational change of the $\beta_{1}-\alpha_{1}$ loop. This shift allows Lys ${ }^{252 *}$ to move out of the active site ('AKR4C7 numbering; Fig. 2B), and facilitates binding of the 5'-phosphoryl group of nicotinamide nucleotide to the enzyme. The new configuration pulls the ribose and nicotinamide rings; which are both then stabilized by residues from Loops A, B and $\beta_{1^{-}}$ $\alpha_{1}$ (Fig. 2B).

We suggest that this role of $\operatorname{Trp}^{21^{*}}$ and $\mathrm{Lys}^{252^{*}}$ in the intermediate-to-tight transition of cofactor binding extends beyond the plant AKR4C7 and AKR4C9 enzymes to include other eukaryotic AKRs. The rationale is based on extensive conservation of 
these key residues in AKRs from protozoa to mammals (Fig. S2). Another event that could contribute to the tight binding of cofactors into AKR4C7 is the attachment of substrate as discussed in the next section.

Substrate binding is likely required to stabilize Loop $B$ in the closed conformation and to induce a catalytically-favorable conformation of Loops $A$ and $C$ in AKR4C7

In contrast with the structures of AKR4C7 reported here, the ternary complex of AKR4C9 shows well-ordered Loops A, B and C, with the Loop B in the closed conformation (Fig. 2A). These comparisons suggest that AKR4C7 requires substrate binding to induce the closed conformation of Loop B and the catalytically-favorable conformation of Loops A and C. In support of this hypothesis, the crystal structure of ternary complexes of AKR4C9 (Fig. 2C) and other AKRs show that the substrate or ligands, such as acetate, contact residues from the Loops A, B and C, likely contributing to their stabilization (Harrison et al., 1994; Simpson et al., 2009; Zheng et al., 2012).

In the AKR4C9 structure, we observed that several residues from Loops A and C contact each other via hydrophobic interactions, contributing to their stabilization in a conformation favouring substrate binding and catalysis. However, in AKR4C7, some of the amino acid substitutions would be predicted to weaken these contacts, a suggestion compatible with disorder of the loops in both apo- and NADP ${ }^{+}$-bound structures (Fig. S3). Since the only apo structure of a plant AKR available thus far (AKR4C1) shows well-ordered Loops $\mathrm{A}$ and $\mathrm{C}$, further studies are required to determine whether substrate-induced structural change is a specific feature of AKR4C7 loops or shared with other plant AKRs such as AKR4C8 and AKR4C9. 
Why are plant AKR4C enzymes poorly active or inactive with glucose and xylose?

Although the closest relatives of the AKR4C subfamily in mammals are members of the AKR1 family, which include aldose reductases, the AKR4C enzymes are poorly active or even inactive with glucose and xylose $[7,9,10]$. We thus postulated that a molecular change to the active site could be responsible for impairment of the xylose/glucose reductase activity in AKR4C subfamily members. To determine whether this was a possibility, we compared the active site of the aldose reductase AKR1B1 (PDB ID: 1ADS) with those of AKR4C9 (PDB ID: 3H7U) and AKR4C8 (PDB ID: 3H7R), which show progressively lower affinity to aldoses (Fig. 3A). Since the impairment of xylose/glucose reductase activity also correlates with a decrease in cofactor affinity, we searched for modifications that would affect both cofactor and substrate binding. Such modifications were found in the intramolecular interactions that hold Loop B in the closed conformation and contribute to shape the substrate-binding site (Fig. 3B-D).

\section{$<$ Figure $3>$}

In AKR1B1, the closed conformation of Loop B is stabilized by two electrostatic interactions connecting $\mathrm{Asp}^{216}$ to $\mathrm{Lys}^{21,262}$ and one hydrophobic interaction linking $\operatorname{Trp}^{219}$ with $\operatorname{Trp}^{20}$. Moreover, the catalytically-favorable positioning of $\operatorname{Trp}^{20}$, which seems to modulate cofactor binding and to play a role in substrate binding, is ensured by a hydrophobic contact with $\mathrm{Tyr}^{48}$ and an aromatic-sulphur interaction with $\mathrm{Cys}^{298}$ (Fig. 3B). The $\mathrm{Cys}^{298}$ residue in particular, is replaced by valine in AKR4C9 and the bulkier leucine in AKR4C7. We suggest that the resulting structural change contributes to the 6-fold higher $K \mathrm{~d}$ for NADPH (AKR4C9) and the increased $K \mathrm{~m}$ for aldoses such as 
xylose (40-fold in AKR4C9 and 78-fold in AKR4C7) (Fig. 3A-C). This hypothesis is supported by mutational studies in AKR1B1 showing that the replacement of Cys ${ }^{298}$ by alanine increases the cofactor $K \mathrm{~d}$ and xylose $K \mathrm{~m}$ to levels approximating those of AKR4C9 and AKR4C7, respectively [23].

In addition to the $\mathrm{C} 298 \mathrm{~V}^{*}$ substitution in AKR4C9 ("AKR1B1 numbering), this enzyme also shows a different mechanism of Loop B closure in which electrostatic interactions are replaced by hydrogen bonds, mostly mediated by water molecules (Fig. 3C). Such modifications could interfere with the kinetics of cofactor and substrate binding. However, to know their exact contribution to the lower aldose affinity of AKR4C9, further mutational and functional studies would be required.

In AKR4C8, which lacks both xylose and glucose reductase activities, the AKR1B1-Cys ${ }^{298}$ residue is conserved. However, the sulphur-aromatic interaction observed in AKR1B1 is lost due to replacement of $\operatorname{Trp}^{20}$ by a tyrosine (Fig. 3D). Moreover, modifications in Loop B partially disrupt the hydrophobic "roof" of the substrate-binding site, likely preventing xylose/glucose stabilization. As a result, the wider substrate-binding site has allowed AKR4C8 to become more efficient with steroid substrates at the expense of losing activity with sugars [7].

In summary, our data show first, the involvement of an intermediate state of cofactor binding in AKR4C7 and suggest a three-step mechanism for NADPH attachment in the enzymatic mechanism of this and other AKRs. Second, our comparative analyses of human AKR1B1 and plant AKR4C9, AKR4C7, and AKR4C8 showed a redesign of the "latches" that stabilize a closed conformation of Loop B (Salt bridges and sulphur-aromatic interactions would be replaced by direct or watermediated H-bonds). We propose that this in turn would affect the cofactor affinity and 
shape of the substrate-binding site, both contributing to progressive impairment of sugar reductase activity in the plant-specific AKR4C subfamily.

\section{Accession numbers}

The structure factors and atomic coordinates of AKR4C7 in the apo form and in complex with $\mathrm{NADP}^{+}$were deposited in the Protein Data Bank repository under the accession codes $5 \mathrm{JH} 1$ and 5JH2, respectively.

\section{Author Contributions}

SMS, JAY and RA designed research, SMS cloned, expressed and purified the recombinant AKR4C7; MLS performed the experiments; POG, MLS, RA and MTM solved and refined the structures; POG, MLS, SMS, JAY, RA and MTM analyzed data; POG and MTM wrote the manuscript; POG, MLS, SMS, KEK, JAY, RA and MTM revised the manuscript. All authors read and approved the final manuscript.

\section{Conflict of interests}

The authors declare no conflicts of interests.

\section{Submission declaration}

The authors declare that this work has not been published previously and is not under consideration for publication elsewhere. The current version of the manuscript was read and approved by all authors. 


\section{Acknowledgements}

This project was funded by grants from the USDA-NRI (2007-35318-18394). We gratefully thank the Brazilian Synchrotron Light Laboratory (LNLS) for the provision of time at the MX2 beamline.

\section{References}

[1] T.M. Penning, The aldo-keto reductases (AKRs): Overview, Chemico-biological interactions, 234 (2015) 236-246.

[2] D. Sengupta, D. Naik, A.R. Reddy, Plant aldo-keto reductases (AKRs) as multitasking soldiers involved in diverse plant metabolic processes and stress defense: A structure-function update, Journal of plant physiology, 179 (2015) 40-55.

[3] J.M. Jez, T.M. Penning, The aldo-keto reductase (AKR) superfamily: an update, Chemico-biological interactions, 130-132 (2001) 499-525.

[4] C.L. Kaul, P. Ramarao, The role of aldose reductase inhibitors in diabetic complications: recent trends, Methods and findings in experimental and clinical pharmacology, 23 (2001) 465-475.

[5] D. Bartels, Targeting detoxification pathways: an efficient approach to obtain plants with multiple stress tolerance?, Trends in plant science, 6 (2001) 284-286.

[6] J.G. Olsen, L. Pedersen, C.L. Christensen, O. Olsen, A. Henriksen, Barley aldose reductase: structure, cofactor binding, and substrate recognition in the aldo/keto reductase 4C family, Proteins, 71 (2008) 1572-1581.

[7] P.J. Simpson, C. Tantitadapitak, A.M. Reed, O.C. Mather, C.M. Bunce, S.A. White, J.P. Ride, Characterization of two novel aldo-keto reductases from Arabidopsis: expression patterns, broad substrate specificity, and an open active-site structure suggest a role in toxicant metabolism following stress, Journal of molecular biology, 392 (2009) 465-480. 
[8] D. Bartels, K. Engelhardt, R. Roncarati, K. Schneider, M. Rotter, F. Salamini, An ABA and GA modulated gene expressed in the barley embryo encodes an aldose reductase related protein, The EMBO journal, 10 (1991) 1037-1043.

[9] R. Roncarati, F. Salamini, D. Bartels, An aldose reductase homologous gene from barley: regulation and function, The Plant journal : for cell and molecular biology, 7 (1995) 809-822.

[10] S.M. de Sousa, L.K. Rosselli, E. Kiyota, J.C. da Silva, G.H. Souza, L.A. Peroni, D.R. Stach-Machado, M.N. Eberlin, A.P. Souza, K.E. Koch, P. Arruda, I.L. Torriani, J.A. Yunes, Structural and kinetic characterization of a maize aldose reductase, Plant Physiol Biochem, 47 (2009) 98-104.

[11] E. Kiyota, S.M. de Sousa, M.L. Dos Santos, A. da Costa Lima, M. Menossi, J.A. Yunes, R. Aparicio, Crystallization and preliminary X-ray diffraction analysis of maize aldose reductase, Acta crystallographica. Section F, Structural biology and crystallization communications, 63 (2007) 990-992.

[12] A.W. Leslie, H. Powell, Processing diffraction data with mosflm, in: R. Read, J. Sussman (Eds.) Evolving Methods for Macromolecular Crystallography, Springer Netherlands2007, pp. 41-51.

[13] W. Kabsch, Evaluation of single-crystal X-ray diffraction data from a positionsensitive detector, Journal of applied crystallography, 21 (1988) 916-924.

[14] M.D. Winn, C.C. Ballard, K.D. Cowtan, E.J. Dodson, P. Emsley, P.R. Evans, R.M. Keegan, E.B. Krissinel, A.G. Leslie, A. McCoy, S.J. McNicholas, G.N. Murshudov, N.S. Pannu, E.A. Potterton, H.R. Powell, R.J. Read, A. Vagin, K.S. Wilson, Overview of the CCP4 suite and current developments, Acta crystallographica. Section D, Biological crystallography, 67 (2011) 235-242. 
[15] R.M. Keegan, M.D. Winn, Automated search-model discovery and preparation for structure solution by molecular replacement, Acta crystallographica. Section D, Biological crystallography, 63 (2007) 447-457.

[16] V. Calderone, B. Chevrier, M. Van Zandt, V. Lamour, E. Howard, A. Poterszman, P. Barth, A. Mitschler, J. Lu, D.M. Dvornik, G. Klebe, O. Kraemer, A.R. Moorman, D. Moras, A. Podjarny, The structure of human aldose reductase bound to the inhibitor IDD384, Acta crystallographica. Section D, Biological crystallography, 56 (2000) 536540.

[17] J. Navaza, AMoRe: an automated package for molecular replacement, Acta Crystallographica Section A, 50 (1994) 157-163.

[18] G.N. Murshudov, A.A. Vagin, E.J. Dodson, Refinement of macromolecular structures by the maximum-likelihood method, Acta crystallographica. Section D, Biological crystallography, 53 (1997) 240-255.

[19] P. Emsley, B. Lohkamp, W.G. Scott, K. Cowtan, Features and development of Coot, Acta crystallographica. Section D, Biological crystallography, 66 (2010) 486-501. [20] V.B. Chen, W.B. Arendall, 3rd, J.J. Headd, D.A. Keedy, R.M. Immormino, G.J. Kapral, L.W. Murray, J.S. Richardson, D.C. Richardson, MolProbity: all-atom structure validation for macromolecular crystallography, Acta crystallographica. Section D, Biological crystallography, 66 (2010) 12-21.

[21] Schrodinger, LLC, The PyMOL Molecular Graphics System, Version 1.3r1, 2010.

[22] G. Sanli, J.I. Dudley, M. Blaber, Structural biology of the aldo-keto reductase family of enzymes: catalysis and cofactor binding, Cell biochemistry and biophysics, 38 (2003) 79-101. 
[23] K.M. Bohren, J.M. Brownlee, A.C. Milne, K.H. Gabbay, D.H. Harrison, The structure of Apo R268A human aldose reductase: hinges and latches that control the kinetic mechanism, Biochimica et biophysica acta, 1748 (2005) 201-212.

[24] W.C. Cooper, Y. Jin, T.M. Penning, Elucidation of a complete kinetic mechanism for a mammalian hydroxysteroid dehydrogenase (HSD) and identification of all enzyme forms on the reaction coordinate: the example of rat liver 3alpha-HSD (AKR1C9), The Journal of biological chemistry, 282 (2007) 33484-33493.

[25] E. Krissinel, K. Henrick, Secondary-structure matching (SSM), a new tool for fast protein structure alignment in three dimensions, Acta crystallographica. Section D, Biological crystallography, 60 (2004) 2256-2268.

[26] K.M. Bohren, J.L. Page, R. Shankar, S.P. Henry, K.H. Gabbay, Expression of human aldose and aldehyde reductases. Site-directed mutagenesis of a critical lysine 262, The Journal of biological chemistry, 266 (1991) 24031-24037.

[27] T.A. Binkowski, S. Naghibzadeh, J. Liang, CASTp: Computed Atlas of Surface Topography of proteins, Nucleic acids research, 31 (2003) 3352-3355. 


\section{Figure Legends}

Figure 1. The overall structure of AKR4C7. Cartoon representation of AKR4C7 structure in (A) the apo form and (B) in complex with $\mathrm{NADP}^{+}$. Catalytic residues are highlighted as labelled sticks with colors to denote Loops A (green), B (violet) and C (pink). Key residues are labeled in A and color-coded in B. Dashed lines represent disordered regions that were not modelled. In panel $B$, the 1,2-ethanediol (EDO) molecule is shown as a green stick for carbon atoms and the adenosine-2'-phosphate moiety of $\mathrm{NADP}^{+}$appears as spheres. The transparent grey oval indicates the binding site for the nicotinamide nucleotide moiety, which was not observed in the electron density map (probably due to high flexibility). Note the change in position and structure of Loop B from (A) to (B).

Figure 2. Conformational changes between the binary complex AKR4C7•NADP+ and the ternary complex AKR4C9・NADP+•acetate. (A) Structural superimposition of $\mathrm{AKR} 4 \mathrm{C} 7 \cdot \mathrm{NADP}+$ onto AKR4C9・NADP+・acetate (PDB ID: $3 \mathrm{H} 7 \mathrm{U}$ ) with highlights showing where Loops A, B and C are partially disordered in AKR4C7 (green dashed lines) and well-structured in AKR4C9 (pink cartoon). The $\beta_{1}-\alpha_{1}$ loop $\left(\operatorname{Ser}^{15}-\mathrm{Gly}^{26}\right.$ ), which displays distinct conformations in AKR4C7 and AKR4C9, is also highlighted 
using the same color scheme. Acetate is shown as solid spheres, whereas 1,2-ethanediol is shown as sticks. While only the adenosine-2'-phosphate moiety of NADP+ is observed in the AKR4C7 structure (green carbon atoms of NADP+), the whole cofactor (pink carbon atoms) is found in the AKR4C9 structure. (B) Close-up view of the AKR4C7 active site and (C) close-up view of the AKR4C9 active site. Note conformational changes required for the full attachment of cofactor in AKR4C7 and likely induced by substrate binding.

Figure 3. Functional and structural comparisons between the human aldose reductase AKR1B1 and the plant AKR4C subfamily members AKR4C7, AKR4C8 and AKR4C9. (A) A summary of affinity parameters for cofactor- and aldose- binding from literature: ${ }^{\mathrm{a}}[23,26],{ }^{\mathrm{b}}[7],{ }^{\mathrm{c}}[10] .(B, C$ and $D)$ Surface topography of the AKR active sites determined using CASTp [27]. The cofactor is shown as spheres, whereas ligands found in the substrate-binding site (dark grey outline in the basal portion of each enzyme) are represented as sticks (green carbon atoms). Amino acid residues that stabilize Loop B in the closed conformation are also shown as sticks. Dashed lines refer to hydrogen bonds or salt bridges, while red spheres indicate water molecules. Below each active site is a simplified scheme of the intra-molecular interactions (lines) that contribute to stabilization of Loop $\mathrm{B}$ in the closed conformation: electrostatic interactions (orange), hydrophobic contacts (beige), aromatic-sulphur interactions (green), side-chain-to-side-chain hydrogen bonds (dark blue) and water mediated hydrogen bonds (light blue). Residues belonging to Loop B are black contoured in the scheme. Residues in the plant AKRs that differ from those of the human enzyme are colored in red. 
Table 1. Summary of X-ray data collection and structure refinement statistics ${ }^{\mathrm{a}}$.

\begin{tabular}{|c|c|c|}
\hline & Apoenzyme & $\mathrm{NADP}^{+}$complex \\
\hline \multicolumn{3}{|l|}{ Data collection } \\
\hline Space group & $P 2{ }_{1} 2_{1}{ }_{1}$ & $P 2{ }_{1} 2_{1}{ }_{1}$ \\
\hline \multicolumn{3}{|l|}{ Cell dimensions } \\
\hline$a, b, c(\AA)$ & $48.22,55.98,103.26$ & $47.22,55.86,105.74$ \\
\hline Resolution $(\AA)^{\mathrm{a}}$ & $37.95-1.45(1.53-1.45)$ & $49.39-1.72(1.81-1.72)$ \\
\hline$R_{\text {merge }}$ & $0.07(0.41)$ & $0.07(0.64)$ \\
\hline$I / \sigma I$ & $13.8(2.8)$ & $11.6(2.0)$ \\
\hline Completeness (\%) & $98.8(97.1)$ & $91.7(74.8)$ \\
\hline Redundancy & $5.2(4.9)$ & $4.8(4.6)$ \\
\hline \multicolumn{3}{|l|}{ Refinement } \\
\hline Resolution $(\AA)$ & $37.95-1.45(1.49-1.45)$ & $49.39-1.72(1.77-1.72)$ \\
\hline No. reflections & $47126(3344)$ & 26401 (1399) \\
\hline$R_{\text {work }} / R_{\text {free }}$ & $0.13 / 0.18(0.31 / 0.34)$ & $0.17 / 0.21(0.35 / 0.37)$ \\
\hline Protein residues & 262 ( 1 chain $)$ & 257 (1 chain) \\
\hline Ligand/ion & 0 & $3(\mathrm{~A} 2 \mathrm{P}, \mathrm{EDO}, \mathrm{CL})$ \\
\hline Water & 307 & 192 \\
\hline \multicolumn{3}{|l|}{$B$-factors average $\left(\AA^{2}\right)$} \\
\hline Protein & 21.24 & 26.88 \\
\hline Ligand/ion & 0 & 29.91 \\
\hline Water & 37.09 & 37.33 \\
\hline \multicolumn{3}{|l|}{ R.m.s. deviations } \\
\hline Bond lengths $(\AA)$ & 0.017 & 0.019 \\
\hline Bond angles $\left(^{\circ}\right)$ & 1.682 & 1.843 \\
\hline \multicolumn{3}{|l|}{ Ramachandran } \\
\hline Favored $(\%)$ & 98.9 & 99.6 \\
\hline Outliers (\%) & 0 & 0 \\
\hline PDB code & 5JH1 & $5 \mathrm{JH} 2$ \\
\hline
\end{tabular}

${ }^{\mathrm{a}}$ Values in parentheses are for highest-resolution shell. 
A

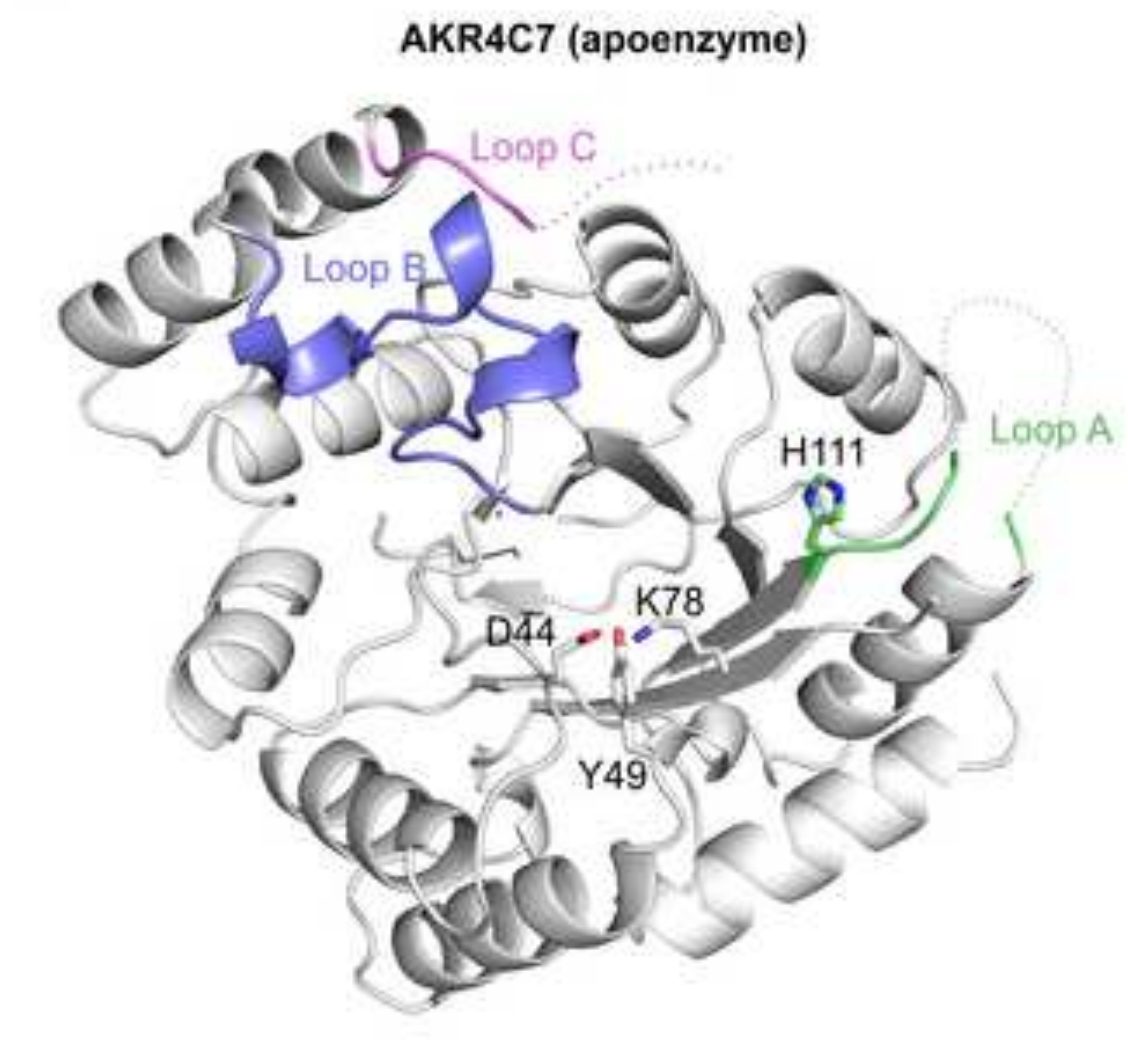

B

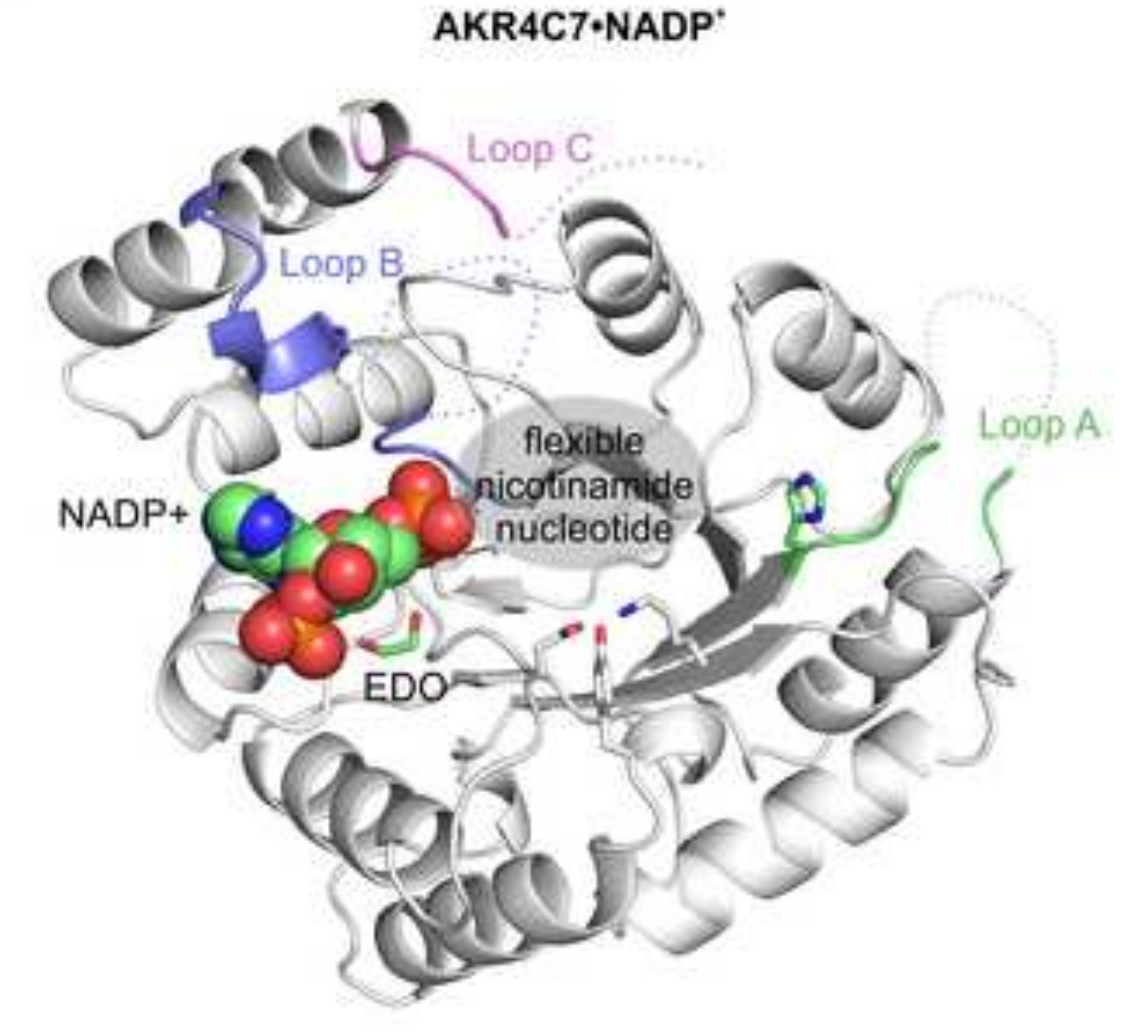


A

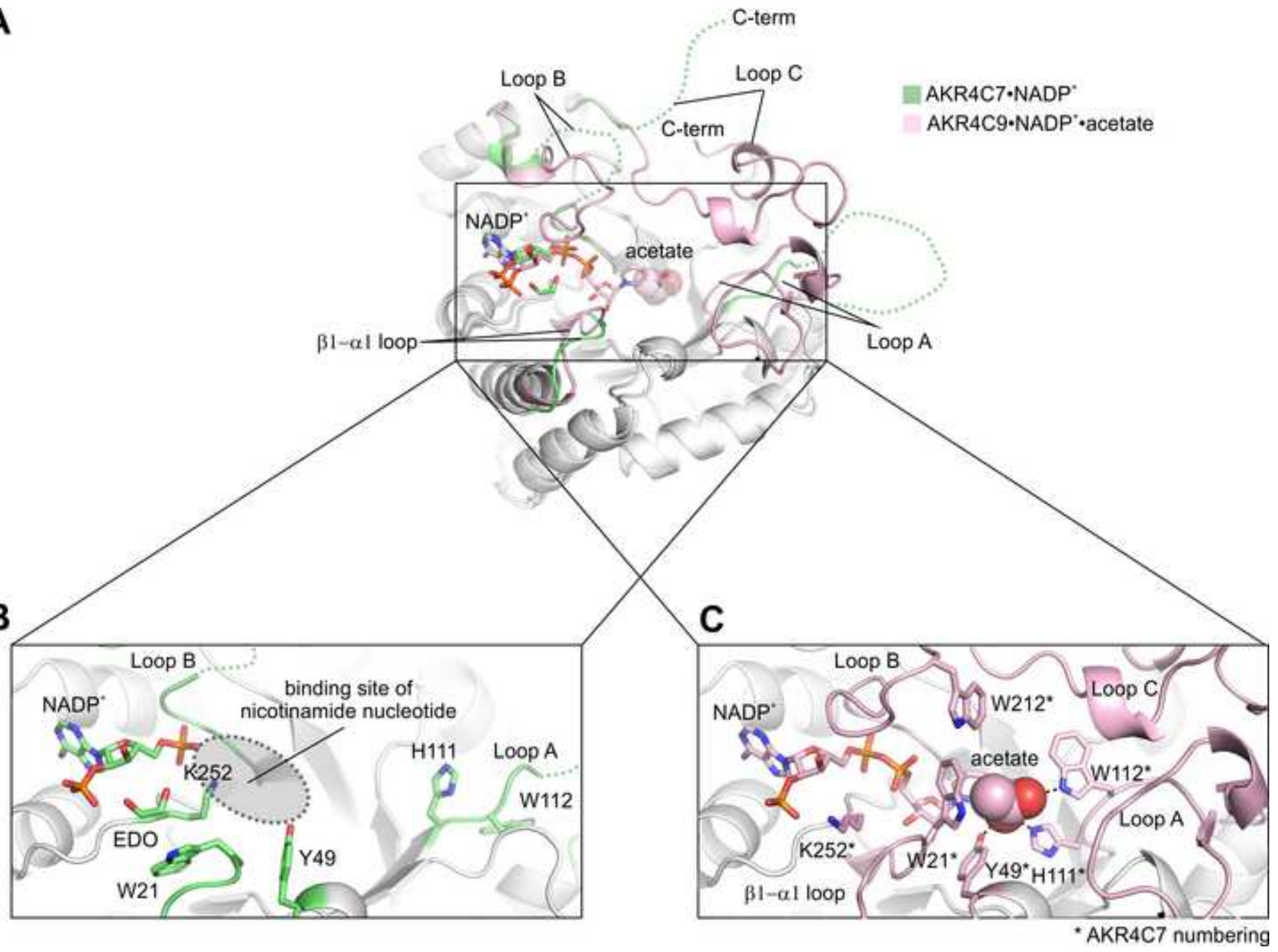


A

\begin{tabular}{|ccccc|}
\hline & $\begin{array}{c}\text { NADPH } \\
\text { Kd (nM) }\end{array}$ & $\begin{array}{c}\text { glyceraldehyde } \\
\text { Km (mM) }\end{array}$ & $\begin{array}{c}\text { xylose } \\
\mathrm{Km}(\mathrm{mM})\end{array}$ & $\begin{array}{c}\text { glucose } \\
\mathrm{Km}(\mathrm{mM})\end{array}$ \\
\hline AKR1B1 $^{*}$ & 10 & 0.02 & 6 & 114 \\
AKR4C9 $^{\circ}$ & 61 & 2.2 & 238 & 760 \\
AKR4C7 $^{\circ}$ & n.d. & 4.3 & 467 & no activity \\
AKR4C8 $^{b}$ & 250 & 22.4 & no activity & no activity \\
\hline
\end{tabular}

B

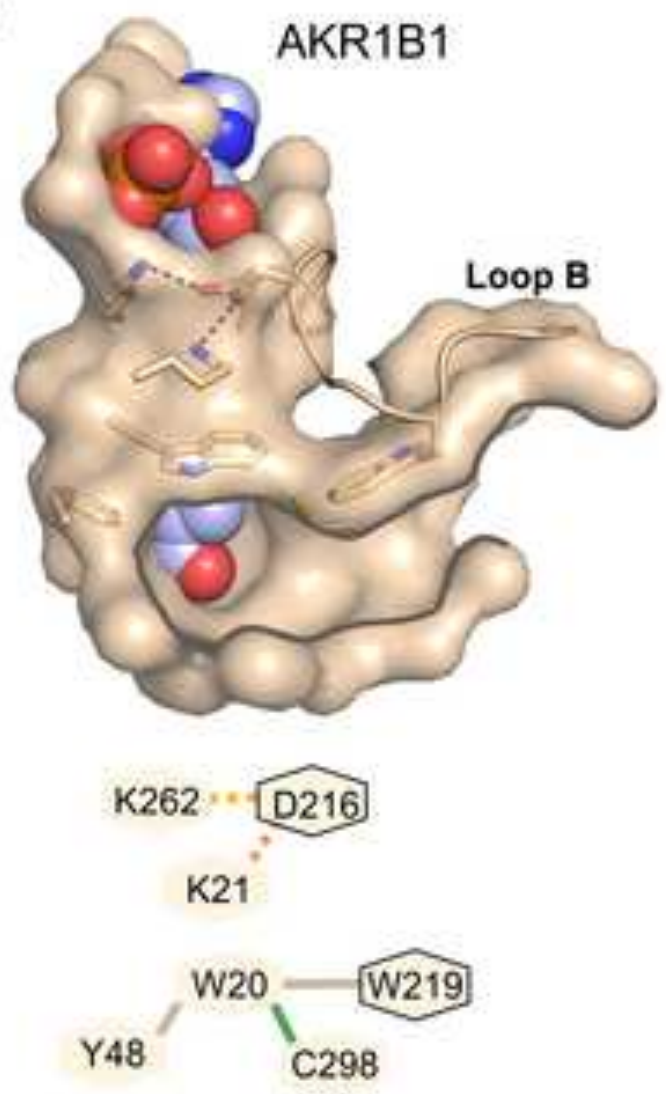

C
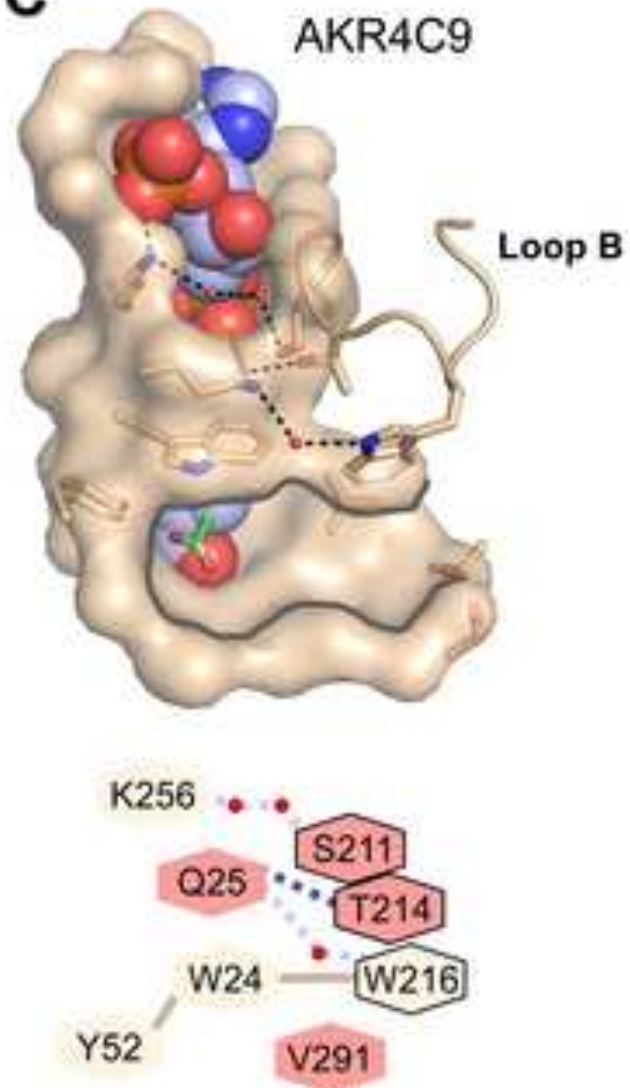

D

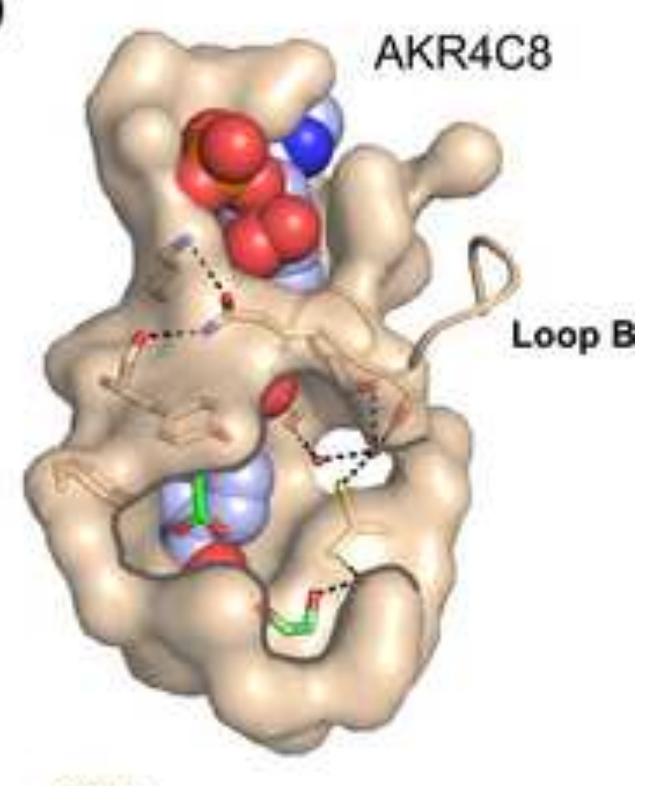

K252.

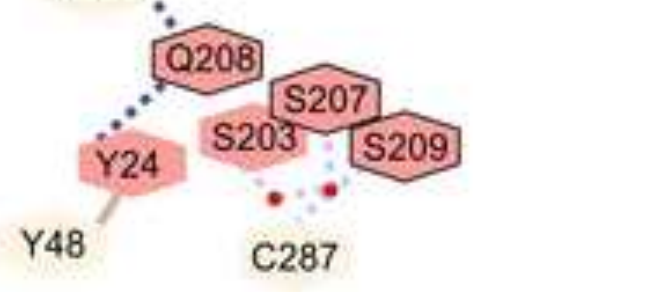

